\title{
The sacral autonomic outflow: against premature oversimplification
}

\author{
Wilfrid Jänig ${ }^{1}$ Elspeth M. McLachlan ${ }^{2}$. Winfried L. Neuhuber ${ }^{3}$
}

Received: 12 December 2017 / Accepted: 14 December 2017 / Published online: 3 January 2018

c) Springer-Verlag GmbH Germany, part of Springer Nature 2018

Using developmental genetic criteria, Espinosa-Medina and co-workers have recently found that lumbar and sacral autonomic pathways show many similarities. Based on these data, they have put forward the idea that the preganglionic and postganglionic neurons of the autonomic nervous system in the sacral spinal cord and in the pelvic ganglia are sympathetic and not parasympathetic [1]. This idea seemingly challenges the traditional view established originally by Langley $[2,3]$ that the peripheral parasympathetic and sympathetic pathways are defined anatomically by the location of the preganglionic neurons in the spinal cord and brain stem. Now Espinosa-Medina and co-workers [2] critically review the arguments put forward more than 100 years ago by Gaskell, Langley and their followers. They come to the conclusion that their "reinterpretation of the sacral outflow in the light of embryonic development [namely as being in fact sympathetic]... [could be] a continuation of Gaskell's and Langley's vision". They conclude furthermore that "Getting rid of the imaginary sympatho-parasympathetic complexity [i.e. generalized antagonistic action] in the [sacral] region will hopefully open the way to deciphering its real complexity: ...".

Although developmental studies can be illuminative, in this case, they show nothing more than the spinal (not "sympathetic") nature of both the thoracolumbar and sacral autonomic pathways. They fall short in failing to decipher the functional differences. We fully concur with the authors that the workings of the autonomic nervous system involved in the regulation of pelvic organs cannot be understood in terms of antagonism between parasympathetic and

Wilfrid Jänig

w.janig@physiologie.uni-kiel.de

1 Physiologisches Institut, Christian-Albrechts-Universität zu Kiel, Kiel, Germany

2 Neuroscience Research Australia, University of New South Wales, Sydney, Australia

3 Institut für Anatomie, Friedrich-Alexander-Universität Erlangen-Nürnberg, Erlangen, Germany sympathetic nervous systems or in terms of homeostatic autonomic regulation [3]. However, we completely disagree with the authors that the sacral autonomic outflow should be labelled "sympathetic". To be clear, we do not question the data of their investigations but the conclusion they have drawn. Their new concept is a premature simplification and would prevent a deeper understanding of the neurobiology of the regulation of pelvic organs. It would bring back the old misconception of that the parasympathetic and sympathetic systems are antagonistic [3] in an even more accentuated way, leading to another myth - that all of the spinal outflow would be activated simultaneously by e.g. fright, fight and flight.

What are our arguments? The autonomic regulation of the pelvic organs is more complex, as far as the final pathways are concerned, than for other autonomic control systems such as the regulation of arterial blood pressure, body temperature, pupil diameter, etc. The minimal number of final autonomic pathways are two sacral ones and one lumbar one for the lower urinary tract [4], one pathway each of the lumbar and sacral autonomic outflows for the hindgut and one sacral pathway and two lumbar pathways for the reproductive organs [3]. On top of these is the lumbar vasoconstrictor pathway to the blood vessels in the pelvic viscera. The three functional pelvic systems each consist of several organs and tissues which operate in distinct ways to perform complex and usually intermittent tasks. Their peripheral pathways are pre- and postganglionically separate, i.e. they do not overlap by anatomical and functional criteria. A suggested minor overlap in the pathways to erectile tissue is in fact functionally distinct in that the sacral vasodilator pathway is activated both reflexly and psychogenically whereas the lumbar vasodilator pathway can only be activated psychogenically [3]. The final autonomic pathways are strictly defined by their function in the target tissue and by the central circuits in spinal cord, brain stem, hypothalamus and telencephalon that are connected to them. Specific functions are reflected in the distinct discharge patterns exhibited by the pre- and postganglionic neurons of each autonomic pathway. These discharge patterns consist of ongoing activity (or lack of it) 
and reflex responses to physiological stimulation of primary afferent neurons (e.g. from the urinary bladder, hindgut, anal canal, penis, arterial baroreceptors or chemoreceptors etc.) $[3,5]$. These discharge patterns provide the functional fingerprints that define each distinct peripheral autonomic pathway. The precise coordination of the activity in these pathways during the regulation of the pelvic organs by the brain is one of the most remarkable and outstanding accomplishments of the nervous system. This coordinated activity can be pressed to fit neither into the parasympathetic-sympathetic (antagonistic) dogma (as if in a procrustean bed) nor into a single sympathetic system. It is clear that the thoracolumbar (sympathetic) and sacral (parasympathetic) autonomic pathways have several distinct functions that complement each other in the regulation of the pelvic organs.

The concept of organised complexity does not apply selectively to the lumbosacral autonomic outflow but may also apply more generally to the function of the autonomic nervous system. Using the terms "sympathetic" and "parasympathetic" in a global sense, as can be inferred from the work of Walter Bradford Cannon and his followers and is still commonly done, has led to generalizations that are not justified in view of the neurobiological differentiation of the autonomic nervous system. Thus, to speak of "parasympathetic function" and "sympathetic function" is misleading and can generate misunderstandings and a wrong impression of how these systems are organized and work [3].

\section{Compliance with ethical standards}

Conflict of interest The authors declare that they have no conflicts of interest.

\section{References}

1. Espinosa-Medina I, Saha O, Boismoreau F, Chettouh Z, Rossi F, Richardson WD, Brunet JF (2016) The sacral autonomic outflow is sympathetic. Science 354:893-897

2. Espinosa-Medina I, Saha O, Boismoreau F, Brunet JF (2017) The "sacral parasympathetic": ontogeny and anatomy of a myth. Clin Auton Res. https://doi.org/10.1007/s10286-017-0478-7

3. Jänig W (2006) The integrative action of the autonomic nervous system: neurobiology of homeostasis. Cambridge University Press, Cambridge

4. De Groat CWC, Griffiths D, Yoshimura N (2015) Neural control of the lower urinary tract. Compr Physiol 5:327-396

5. Jänig W, McLachlan EM (1987) Organization of lumbar spinal outflow to distal colon and pelvic organs. Physiol Rev 67:1332-1404 